\title{
Clinical Profile and Outcome of Children with Acute Central Nervous System Infection in Kerala, India
}

\author{
Authors: \\ *Mili Thomas, ${ }^{1}$ Kamala Swarnam, ${ }^{1}$ Indu Sunitha Viswanathan, ${ }^{1}$ Gopika \\ Sekhar Remadevi, ${ }^{1}$ Nazeer Khan, ${ }^{1}$ T. V. Anilkumar ${ }^{2}$ \\ 1. Department of Pediatrics, Ananthapuri Hospitals and Research Institute, \\ Thiruvananthapuram, India \\ 2. Department of Neurology, Ananthapuri Hospitals and Research Institute, \\ Thiruvananthapuram, India \\ *Correspondence to militreesa@gmail.com \\ Disclosure: $\quad$ The authors have declared no conflicts of interest. \\ Received: $\quad 18.04 .20$ \\ Accepted: $\quad 15.06 .20$ \\ Keywords: $\quad$ Acute central nervous system (CNS) infection, children, clinical profile, encephalitis, \\ meningitis, meningococcus, pneumococcus, scrub typhus. \\ Citation: \\ EMJ. 2020;5[4]:96-104
}

\section{Abstract}

Background and aims: Infection of the central nervous system is a significant cause of morbidity and mortality in children. The aim of this study was to evaluate clinical profile and outcome of children aged 1 month to 15 years admitted with acute central nervous system infection between 2008 and 2020 in the Department of Pediatrics, Ananthapuri Hospitals and Research Institute, Thiruvananthapuram, India.

Materials and methods: This was a case record based retrospective study.

Results: Of 62 children, 44 had meningitis and 18 had encephalitis. Most patients were in the age group 1-5 years old and males were the predominant sex (70.96\%). Eighteen patients with meningitis had a clinical triad of fever, headache, and vomiting, while only three with encephalitis experienced this. Seizures and altered sensorium were seen significantly more in children with encephalitis. Cerebrospinal fluid pleocytosis was seen in significantly more patients with meningitis compared with patients with encephalitis. Aetiology for meningitis included pneumococcus, Orientia tsutsugamushi (scrub typhus), meningococcus, and Angiostrongylus cantonensis infection. Causes of encephalitis included enterovirus, mumps virus, herpes simplex virus, dengue virus, and H1N1 influenza virus infection. Paediatric intensive care unit admission was more common for patients with encephalitis. One child with pneumococcal meningitis and another with dengue encephalitis died. Seizures were the most common sequelae.

Conclusion: Typical clinical features were not present in most patients with meningitis; therefore, a high index of suspicion is needed for early diagnosis. Exact aetiologies could not be identified in most of the patients. Pneumococcus, scrub typhus, and meningococcus were the aetiological agents identified for meningitis. Encephalitis was attributed to dengue virus, herpes simplex virus, enterovirus, mumps virus, and H1N1 influenza virus infection. 


\section{INTRODUCTION}

Infection of the central nervous system (CNS) is a significant cause of morbidity and mortality in children. Depending on the tissues affected, infection could result in meningitis, encephalitis, meningoencephalitis, or a brain abscess.' Globally, the incident cases of meningitis increased from 2.50 million (95\% uncertainty interval: 2.192.91 ) in 1990 to 2.82 million (95\% uncertainty interval: 2.46-3.31) in 2016, but the global number of meningitis deaths was estimated to have decreased by 21\% from 403,012 in 1990 to 318,400 in $2016 .{ }^{2}$ In India, the incidence and age standardised rate of meningitis have decreased by $51.7 \%$ and $28.1 \%$, respectively, from 1990 to $2016 .{ }^{2}$ This was achieved by the universal immunisation programmes against major pathogens, such as Haemophilus influenzae Type b, Streptococcus pneumoniae, and Neisseria meningitidis. Acute encephalitis, defined as the acute onset of fever, a change in mental status, and/or new onset of seizures (excluding simple febrile seizures), is clearly a pressing public health emergency in India. Recurrent epidemics of encephalitis of unknown aetiology have occurred in the country. ${ }^{3}$

Kerala, India, experiences a tropical climate, as it is located 800 miles from the equator. ${ }^{4}$ The advancements in healthcare in Kerala are on par with developed countries and the country achieves among the best health outcomes with a fairly robust primary healthcare system compared with other states in India. Every village panchayat (village council) has a primary healthcare centre; there is a subcentre for every 5,000 people, which is standard for India. Approximately $60-70 \%$ of primary care services are provided in private hospitals. Some patients are referred to hospitals from subcentres, but most people seek care directly from major hospitals. ${ }^{5}$ In 2019, there were 59 reported cases and five deaths from acute encephalitis syndrome in Kerala. Of these cases, 11 diagnoses and two deaths were caused by Japanese encephalitis. ${ }^{6}$

This study evaluated the demographic, clinical, and radiological profiles, and outcome of acute CNS infection in children aged 1 month to 15 years admitted to the Department of Pediatrics, Ananthapuri Hospitals and Research Institute in Kerala, India, between 2008 and 2020.

\section{MATERIALS AND METHODS}

This was a case record based retrospective study. Case records of all patients aged 1 month to 15 years who were admitted with acute CNS infection to the hospital between 2008 and 2020 were reviewed. Demographic data, clinical features, radiological features, course during hospital stay, and follow-up data were recorded using a structured pro forma. Inclusion criteria were ages 1 month to 15 years, files having complete data, and conclusive diagnosis of acute CNS infection. Case files with incomplete data, noninfective aetiologies, and age group $<1$ month and $>15$ years were excluded. The diagnosis of each patient was revised using clinical features and lab evidences. Automated complete blood counts were done using the Coulter principle. Differential counts were also rechecked manually. Absolute neutrophil count (ANC) $>10,000 / \mathrm{mm}^{3}$ was considered as neutrophilic leukocytosis and absolute lymphocyte count $>8,000 / \mathrm{mm}^{3}$ was considered as lymphocytic leukocytosis. Erythrocyte sedimentation rate (ESR) was determined using Westergrens principle. C-reactive protein (CRP) was measured using immuno-microslide assays. ESR $>40 \mathrm{~mm} / \mathrm{hr}$ and CRP $>30 \mathrm{mg} / \mathrm{L}$ were considered positive. Cerebrospinal fluid (CSF) was centrifuged at 3,000 rpm and checked under light microscopy for cytology. CSF gram staining was carried out in all patients. CSF protein and sugar were tested with the turbulometric method. CSF protein $>80 \mathrm{mg} / \mathrm{dL}$ was considered to be elevated, though age-dependent variations in normal value were present. CSF glucose $<45 \mathrm{mg} / \mathrm{dL}$ or CSF blood-glucose ratio $<0.6$ were considered as hypoglycorrhachia. Blood and CSF cultures were performed using the BACTEC ${ }^{\mathrm{TM}}$ method (Becton, Dickinson and Company, Franklin, New Jersey, USA). CSF multiplex PCR, brain MRI, or electroencephalogram was carried out for some patients. Reports of radiological investigations were analysed and classified as features of encephalitis, meningitis, or both.

Patients with clinical features of meningitis with ANC $>10,000 / \mathrm{mm}^{3}$, elevated ESR and CRP, and CSF neutrophilic pleocytosis with or without elevated protein and hypoglycorrhachia were classified as having bacterial meningitis. Patients with CSF gram stain showing bacteria with sterile cultures were also considered 
as having bacterial meningitis. Patients with clinical features of meningitis without clear evidence of bacterial meningitis from laboratory findings and who received oral or parenteral antibiotics prior to admission were considered as partially-treated meningitis. Patients with clinical features of meningitis with normal ANC and CSF lymphocytic pleocytosis without prior antibiotic administration were considered as having viral or aseptic meningitis. Any pathogen identified in CSF PCR was considered as the aetiological agent. Patients with hemiplegia and or cranial nerve palsy along with radiological features or laboratory evidence with or without strong contact history were considered as tuberculous meningitis, given the high incidence in India. Patients with fever and altered sensorium with or without seizures and without alternative diagnosis were considered as having encephalitis. Patients with radiological features of encephalitis in MRI brain scans were also included in this group. Telephone interviews with all patients were carried out to record the sequelae of the disease. SPSS ${ }^{\circledR}$ Statistics V22.0 (IBM, Endicott, New York, USA) was used to analyse the results. Chi-square test and Fisher's exact test were used to determine the level of significance. Percentages and averages were also calculated. Institutional ethical committee approval was obtained prior to the study.

\section{RESULTS}

Sixty-two children with acute CNS infection were enrolled into the study. Forty-three patients had meningitis, 17 patients had encephalitis, one had meningoencephalitis, and one had cerebral abscess. For the ease of analysis, cerebral abscess was included in meningitis group and meningoencephalitis was included in encephalitis group. There were 27 patients $(43.54 \%)$ in the age group 1-5 years and 16 patients in the infant (1 month-1 year old) group (25.80\%); together, they constituted $69.34 \%$ of the study population. There were 44 males (70.96\%) enrolled in the study and this was the predominant sex. Similar age and sex distributions were seen in patients with encephalitis or meningitis. Twenty-nine (46.77\%) patients received either oral (nine) or parenteral (20) antibiotics before admission. The most common antibiotic used was intravenous ceftriaxone (7) followed by amikacin (5), the most common oral antibiotic used was cefixime (3), and intravenous acyclovir was used in five patients before admission. Comorbidities contributing to acute CNS infection were seen in seven (15.90\%) patients with meningitis (Figure $1 \mathrm{~A}$ and $1 \mathrm{~B}$ ).

There were 18 (40.90\%) patients with meningitis who had a clinical triad of fever, headache, and vomiting; three patients (16.66\%) with encephalitis experienced this, but the difference was not statistically significant $(p=0.082)$. All patients with encephalitis had fever with any of the neurological symptoms. The most common neurological symptom was seizures experienced by 14 patients (77.77\%) followed by altered sensorium experienced by 11 patients (61.11\%). Neurological symptoms were present in 21 patients (47.72\%) with meningitis. Irritability, experienced by 14 patients (31.8\%), was the most common neurological symptom in the meningitis group followed by seizures, experienced by 10 patients $(22.72 \%)$. Seizures $(p<0.001)$ and altered sensorium $(p=0.002)$ were seen significantly more in children with encephalitis (Table 1).

The most common clinical sign seen in patients with meningitis was neck stiffness (10/44 [22.72\%]) followed by low Glasgow Coma Scale (GCS) (9/44 [20.45\%]). In patients with encephalitis, the most common clinical sign was low GCS (10/18 [55.55\%]) followed by motor abnormalities (4/18 [22.22\%]). Of all the clinical signs, low GCS was seen significantly more in children with encephalitis (10/18; $p=0.006$ ) (Table 1). ANC $>10,000 / \mathrm{mm}^{3}$ was seen in 19 (43.18\%) patients with meningitis and seven (38.88\%) patients with encephalitis. Peripheral eosinophilia was seen in one patient with eosinophilic meningitis. Elevated CRP was more significant in patients with meningitis compared with encephalitis $(p=0.025)$. Blood culture was sterile in all patients (Table 1).

CSF pleocytosis was seen in 41 (93.18\%) patients with meningitis compared with nine (50\%) patients with encephalitis $(p<0.001)$. Most patients with meningitis had lymphocytic pleocytosis in CSF (35/44 [79.55\%]). Neutrophilic pleocytosis was only seen in six (13.63\%) patients. CSF protein levels were elevated in 14 (31.81\%) patients with meningitis and one patient with encephalitis $(p=0.049)$ (Table 1). 


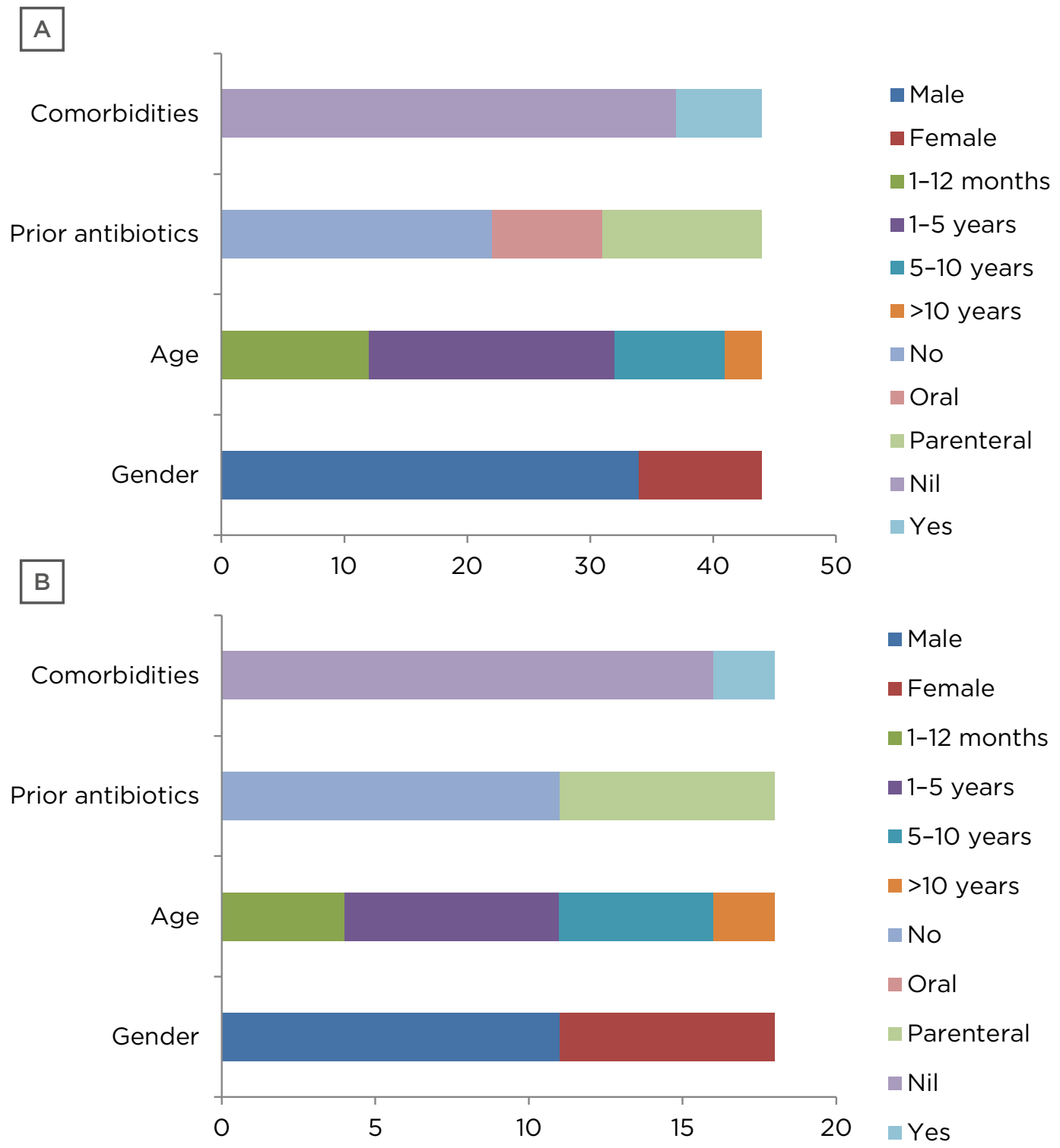

Figure 1: Demographic profile of $\mathbf{A}$ ) meningitis group and $\mathbf{B}$ ) encephalitis group.

A CSF gram stain showed gram positive cocci in two patients, but the culture was sterile. The CSF culture grew penicillin-sensitive S. pneumoniae in one patient. CSF PCR showed S. pneumoniae (one patient), N. meningitidis (one patient), Angiostrongylus cantonensis (one patient), enterovirus (one patient), and herpes simplex virus (one patient).

MRI of the brain was completed for 34/62 (59.67\%) patients, 17 (50\%) of whom had normal results. Eight patients with meningitis and nine patients with encephalitis had positive MRI findings $(p=0.008)$ (Table 1$)$. Two patients with meningitis had basal ganglia involvement and one had mumps encephalitis. One patient with encephalitis was found to have acute necrotising encephalopathy of childhood caused by H1N1 influenza virus infection. One patient had multiple cerebral abscesses. Electroencephalogram was abnormal in 15 (83.33\%) patients with encephalitis and two (11.11\%) patients experienced nonconvulsive status epilepticus.

Twenty-six (59.09\%) patients with meningitis and 17 (94.40\%) patients with encephalitis were admitted to the paediatric intensive care unit $(p=0.012)$. Five (8.06\%) patients required invasive ventilation. 
Table 1: Clinical features and investigations of the study population.

\begin{tabular}{|c|c|c|c|c|}
\hline & \multicolumn{2}{|c|}{ Meningitis $(n=44)$} & \multicolumn{2}{|c|}{ Encephalitis $(n=18)$} \\
\hline & $\mathrm{n}$ & Percentage (\%) & $n$ & Percentage (\%) \\
\hline $\begin{array}{l}\text { Triad of fever, headache, and } \\
\text { vomiting }\end{array}$ & 18 & 40.90 & 3 & 16.67 \\
\hline Seizures* & 10 & 22.72 & 14 & 77.78 \\
\hline Irritability & 14 & 31.80 & 6 & 33.33 \\
\hline GCS $<15^{*}$ & 9 & 20.45 & 10 & 55.56 \\
\hline Motor abnormalities & 4 & 9.09 & 4 & 22.22 \\
\hline Cranial nerve palsy & 1 & 2.27 & 0 & 0.00 \\
\hline Meningeal signs & 10 & 22.72 & 2 & 11.11 \\
\hline Bulging AF & 3 & 6.81 & 0 & 0.00 \\
\hline \multicolumn{5}{|l|}{ Blood count } \\
\hline Normal & 15 & 34.09 & 9 & 50.00 \\
\hline $\begin{array}{l}\text { Neutrophilic leukocytosis } \\
\left(\text { ANC }>10,000 / \mathrm{mm}^{3}\right)\end{array}$ & 19 & 43.18 & 7 & 38.89 \\
\hline $\begin{array}{l}\text { Lymphocytic leukocytosis } \\
\left(\mathrm{ALC}>8,000 / \mathrm{mm}^{3}\right)\end{array}$ & 9 & 20.45 & 2 & 11.11 \\
\hline Eosinophilia & 1 & 2.27 & 0 & 0.00 \\
\hline \multicolumn{5}{|l|}{ ESR } \\
\hline Negative & 25 & 56.81 & 16 & 88.89 \\
\hline$>40$ & 15 & 34.09 & 2 & 11.11 \\
\hline$>100$ & 4 & 9.09 & 0 & 0.00 \\
\hline \multicolumn{5}{|l|}{ CRP } \\
\hline Negative $<30$ & 30 & 68.18 & 16 & 88.89 \\
\hline Positive $>30^{*}$ & 14 & 31.81 & 2 & 11.11 \\
\hline \multicolumn{5}{|l|}{ CSF cytology* } \\
\hline Normal & 2 & 4.54 & 9 & 50.00 \\
\hline Neutrophilic pleocytosis & 6 & 13.63 & 2 & 11.11 \\
\hline Lymphocytic pleocytosis & 35 & 79.55 & 7 & 38.89 \\
\hline \multicolumn{5}{|l|}{ CSF protein } \\
\hline Normal & 30 & 68.18 & 17 & 94.44 \\
\hline Increased* & 14 & 31.81 & 1 & 5.56 \\
\hline \multicolumn{5}{|l|}{ Hypoglycorrhachia } \\
\hline Present & 25 & 56.81 & 7 & 38.89 \\
\hline Absent & 19 & 43.18 & 11 & 61.11 \\
\hline \multicolumn{5}{|l|}{ Brain MRI } \\
\hline Not done & 25 & 56.81 & 3 & 16.67 \\
\hline Positive* & 8 & 18.18 & 9 & 50.00 \\
\hline Negative & 11 & 25.00 & 6 & 33.33 \\
\hline
\end{tabular}

${ }^{*} p<0.05$

AF: anterior fontanelle; ANC: absolute neutrophil count; ALC: absolute lymphocyte count; CRP: C-reactive protein;

CSF: cerebrospinal fluid; GSC: Glasgow Coma Scale; ESR: erythrocyte sedimentation rate. 
For most patients $(77.28 \%$ with meningitis and $94.44 \%$ with encephalitis), first-line antibiotics, such as ceftriaxone or cefotaxime with vancomycin, were started at admission. In other patients, second-line antibiotics, such as piperacillin/tazobactam or meropenem, were used. Antibiotics were upgraded for seven patients with meningitis and for two patients with encephalitis. Acyclovir was used empirically in 20 (45.45\%) patients with meningitis and all patients with encephalitis. Other treatments given in both groups are shown in Table 2. Mean duration of antibiotic treatment was 8.18 days for meningitis and 7.44 days for encephalitis, and mean duration of hospital stay was 11.97 and 19.83 days, respectively.
Exact aetiology was only identified in 11 (17.7\%) patients. Six (13.95\%) patients with meningitis had acute bacterial meningitis and 18 (41.86\%) had partially-treated meningitis; eight (18.60\%) patients had viral meningitis. A diagnosis of tuberculous meningitis was made for four patients based on clinical features, including cranial nerve palsy or hemiplegia; radiological features, such as basal meningitis with vasculitis; and contact history; evidence of tuberculous infection could not be obtained. The patients were treated with antituberculous drugs. Two children had pneumococcal meningitis and they were both $>5$ years old. One child had ventriculoperitoneal shunt in situ and survived. The other child had fulminant presentation of septic shock and cardiac dysfunction. Other aetiological agents identified are given in Table 2.

Table 2: Treatment of study population.

\begin{tabular}{|c|c|c|c|c|}
\hline & \multicolumn{2}{|c|}{ Meningitis $(n=44)$} & \multicolumn{2}{|c|}{ Encephalitis $(n=18)$} \\
\hline & $n$ & Percentage (\%) & $n$ & Percentage (\%) \\
\hline PICU admission* & 26 & 59.09 & 17 & 94.44 \\
\hline \multicolumn{5}{|l|}{ Initial antibiotics } \\
\hline First-line antibiotics & 34 & 77.27 & 17 & 94.44 \\
\hline Second-line antibiotics & 10 & 22.72 & 1 & 5.56 \\
\hline Upgradation of antibiotics & 7 & 15.90 & 2 & 11.11 \\
\hline Steroids & 9 & 20.45 & 5 & 27.78 \\
\hline Acyclovir & 20 & 45.45 & 18 & 100.00 \\
\hline Antiepileptics & 10 & 22.72 & 17 & 94.44 \\
\hline Mannitol & 10 & 22.72 & 4 & 22.22 \\
\hline Invasive ventilation & 2 & 4.54 & 2 & 11.11 \\
\hline Antituberculous treatment & 4 & 9.09 & 0 & 0.00 \\
\hline IVIG & 0 & 0.00 & 2 & 11.11 \\
\hline Plasmapheresis & 0 & 0.00 & 1 & 5.56 \\
\hline Oseltamivir & 1 & 2.27 & 1 & 5.56 \\
\hline
\end{tabular}

IVIG: intravenous immunoglobulin; PICU: paediatric intensive care unit. 
Complications were seen in eight (12.90\%) patients. Septic shock was the most common complication (three patients), followed by acute kidney injury (two patients), nonconvulsive status epilepticus (two patients), catheter-related urinary tract infection (two patients), aspiration pneumonia (two patients), status epilepticus (one patient), and cardiac dysfunction (one patient). Two (3.22\%) patients died as a result of acute CNS infection: one had pneumococcal meningitis with multiorgan dysfunction and the other patient had dengue encephalitis with super refractory seizures. Overall case fatality rates for acute CNS infection in this study was 3.2\%, 2.3\% for meningitis, and 5.5\% for encephalitis.

Follow-up over the telephone was completed for 39 patients. Seven patients (38.88\%) with encephalitis had sequelae. Seizures needing continuation of antiepileptic medications was the most common sequelae and was required by four patients, and poor scholastic performance was reported in two patients. Other sequelae reported included developmental delay, aphasia, motor abnormalities, and dystonia. One patient with meningitis had hearing difficulties.

\section{DISCUSSION}

In this study, most children were $<5$ years of age (70.96\%). Males were the predominant sex in the study group (70.96\%) and the male-female ratio was 2.44. In a study by Debnath et al. ${ }^{7}$ in Pune, India, in children $<12$ years of age, $74.68 \%$ were $<5$ years of age; $46.8 \%$ were infants, $27.9 \%$ were 1-5 years old, and male-female ratio was $1.82 .^{7}$ In a similar study by Mani et al. ${ }^{8}$ from 1996 to 2005, the male-female ratio was $3.18 .^{8}$

Typical clinical features were not present in all children. In this study, less than one-half of patients with meningitis had the triad of fever, headache, and vomiting. Only approximately onehalf of the patients had neurological symptoms. Irritability was the most common neurological symptom in patients with meningitis. A similar demographic was seen in a study by Chauhan et al. ${ }^{9}$ In the 2009 systematic review by Curtis et al., ${ }^{10}$ a lack of irritability lowered the odds of the meningitis by one-half, but the presence of irritability did not strongly signify the presence of meningitis. ${ }^{10}$ Only $25.5 \%$ of patients with meningitis had meningeal signs, similar to results seen in the study by Chinchankar et al." A high index of suspicion is needed to identify children with meningitis early. All patients with encephalitis had fever with neurological symptoms, seizures being the most common. More seizures and altered sensorium were observed in patients with encephalitis, which was expected. In a study by Tripathy et al., ${ }^{12}$ low GCS was more significantly observed in patients with viral encephalitis compared with nonviral cases. ${ }^{12}$

Elevated CRP, CSF pleocytosis, and elevated CSF protein were significantly higher in patients with meningitis. In the study by Fitzwater et al., ${ }^{13}$ elevated CSF white blood cell counts and protein levels were significantly seen more often in patients with bacterial meningitis than in aseptic meningitis. ${ }^{13}$

Pneumococcus sensitive to penicillin grew in CSF culture for one (1.6\%) patient. In a study by Chauhan et al., ${ }^{9}$ CSF culture positivity was seen in $2.0 \%$ of patients. ${ }^{9}$ Higher CSF culture positivity can be seen in other studies, such as those by Debnath et al., ${ }^{7}$ Mani et al., ${ }^{8}$ and De et al. ${ }^{14}$ The low CSF culture positivity seen in this study could be attributed to the approximate one-half of patients having received antibiotics prior to hospital admission, as seen in the study by De et al. ${ }^{14}$ CSF PCR was only completed for 28 (45.16\%) patients, due to financial constraints. Antigen detection tests were not done in this study, which led to failures in exact aetiological diagnosis in many patients.

Admission to the paediatric intensive care unit was needed significantly more by patients with encephalitis. There were only four patients who needed invasive ventilation, and all were for poor sensorium; most of these patients responded to first-line antibiotics. Second-line antibiotics were given at admission in patients with fulminant presentation and for those who were treated with first-line antibiotics externally. Acyclovir was started empirically in approximately onehalf of the patients with meningitis because of an atypical CSF picture and low culture positivity rate.

In India, vaccination against $H$. influenzae Type $b$ has been part of the national immunisation schedule since 2011. Pneumococcal vaccination is part of national immunisation schedule in some states in India. In Kerala, the authors' state, 
it is not part of the government immunisation schedule but is available privately. In the Indian Council of Medical Research (ICMR) study by Jayaraman et al. ${ }^{15}$ in 2012-2013, the most common aetiological agents for meningitis were S. pneumoniae (82.9\%), H. influenzae Type b $(14.4 \%)$, and $N$. meningitidis $(2.7 \%)$. In this present study, two children had pneumococcal meningitis and one with fulminant presentation died on the second day after admission to hospital. This emphasises the importance of pneumococcal vaccination in children. One child with meningococcal meningitis survived without sequelae.

Scrub typhus IgM was positive for two patients with meningitis who had persistent fever. Scrub typhus meningitis, a disease endemic to this part of India, is an important cause of acute and subacute meningitis. Characteristic eschar is seen in some children. ${ }^{16}$ CSF findings include mild pleocytosis, mildly elevated protein, and normal glucose..$^{7,18}$ In a study by Dinesh Kumar et al., ${ }^{19}$ the incidence of meningoencephalitis was $5 \%$ in children with scrub typhus and was commonly seen in ages 5-12 years old, presenting late in the second week of illness during the post-monsoon months. Most of the children responded well to doxycycline. ${ }^{19}$

In this present study, one patient had eosinophilic meningitis, which is a rare entity, and was shown to be caused by $A$. cantonensis. It is characterised by CSF eosinophilia, mildly elevated protein, and normal glucose. ${ }^{20}$ This patient had exposure to snails at home, had peripheral as well as CSF eosinophilia, and typical CSF findings. CSF PCR was positive for $A$. cantonensis. The patient was treated with steroids and albendazole, and recovered without complications.

While most studies across 1975-1999 identified the Japanese encephalitis virus as the main cause of acute encephalitis syndrome, many studies published after the year 2000 identified Chandipura vesiculovirus and enteroviruses as the most common agents for the syndrome, in both outbreak and surveillance studies. ${ }^{21}$ The most common aetiological agent of viral encephalitis in the study by Beig et al. ${ }^{22}$ was enterovirus 71, which occurred in $42.1 \%$ of cases, followed by measles in $21.1 \%$, varicella-zoster virus in $15.8 \%$, herpes simplex virus in $10.5 \%$, and mumps in
$10.5 \%$ of cases; Japanese encephalitis virus was not found in any case. ${ }^{22}$ In a study by Tripathy et al., ${ }^{12}$ herpes simplex virus was the most common cause of viral encephalitis. ${ }^{12}$ In a study by Kumar et al., ${ }^{23}$ herpes simplex virus (31.50\%) was the most common virus followed by adenovirus (10.95\%), parvovirus (2.73\%), Japanese encephalitis virus (1.36\%), enterovirus (1.36\%), and Epstein-Barr virus $(1.36 \%)$.

In this study, exact aetiology was identified in only five of the 18 patients with encephalitis. Japanese encephalitis virus was not identified. One child with dengue encephalitis had super refractory seizures and died. Other aetiologies were herpes simplex virus, enterovirus, mumps virus, and H1N1 influenza virus.

Overall, case fatality rate for acute CNS infection in this study was $3.2 \%$; case fatality rate was $2.3 \%$ for meningitis and 5.5\% for encephalitis. Higher case fatality rate is seen in similar studies. ${ }^{711}$ In this study, the low rate may be attributable to the small sample size. Almost similar complications and neurological sequelae are seen in similar studies. ${ }^{7,11}$

There are several limitations for this study, which include small sample size and the retrospective nature of the study. Audiology evaluation was not done in most patients, hence data on hearing loss was not available. The hospital is a tertiary care referral centre and patients were from neighbouring districts and states with many seeing nearby pediatricians or neurologists for follow-up; therefore, follow-up data after discharge were not available for all patients and only telephone interviews with the parents were possible. As this hospital is frequented by people with middle and high socioeconomic status, the results may not represent the general population. Lack of antigen detection studies and CSF PCR for all children affected the aetiological diagnoses.

\section{CONCLUSION}

Children $<5$ years of age and male sex were predominantly affected in this study. Typical clinical features were not present in most patients with meningitis; therefore, a high index of suspicion is needed for early diagnosis. Seizures, altered sensorium, and need for intensive care 
admission were observed in greater numbers in patients with encephalitis. Elevated CRP, CSF pleocytosis, and elevated CSF protein levels were significantly higher in patients with meningitis compared with patients with encephalitis. For most patients, the aetiological agent could not be identified. Pneumococcus, scrub typhus, and meningococcus were aetiological agents identified for meningitis. Aetiological agents identified for encephalitis were enterovirus, herpes simplex virus, mumps virus, and H1N1 influenza virus. One child died from pneumococcal meningitis and one child died from dengue encephalitis. The most common postencephalitis complications included seizures requiring continued antiepileptic medications.

\section{References}

1. Janowski A, Hunstad D. "Central nervous system infections," Kliegman $\mathrm{R}$ et al. (eds.), Nelson Textbook of Pediatrics (2020), 21 $1^{\text {st }}$ ed. Philadelphia: Elsevier, pp. 2904.

2. Zunt J et al. Global, regional, and national burden of meningitis, 19902016: a systematic analysis for the Global Burden of Disease Study 2016. Lancet Neurol. 2018;17(12):1061-82.

3. Narain J, Lal S. Responding to the challenge of acute encephalitis syndrome/JE in India. J Commun Dis. 2014;46(1):1-3.

4. Government of Kerala, Official Web Portal. About Kerala. 2018. Available at: https://kerala.gov.in/about-kerala. Last accessed: 30 May 2020.

5. Park K. Park's Textbook of Preventive and Social Medicine, 2015. 25 th ed. Jabal-pur: Bhanot Publishers; 2019.

6. Directorate of Health Services. Data on communicable diseases. Available at: http://dhs.kerala.gov.in/dataon-communicable-diseases/. Last accessed: 30 May 2020.

7. Debnath D et al. Epidemiological study of acute bacterial meningitis in admitted children below twelve years of age in a tertiary care teaching hospital in Pune, India. Med J DY Pati Univ. 2012;5(1):28-30.

8. Mani R et al. Bacteriological profile of community acquired acute bacterial men-ingitis: a ten-year retrospective study in a tertiary neurocare centre in South India. Indian J Med Microbiol. 2007;25(2):108-14.
9. Chauhan D et al. Epidemiology, clinical profile and role of rapid tests in the diagnosis of acute bacterial meningitis in children (aged 1-59 months). Neurol India. 2018;66(4):1045-49.

10. Curtis $\mathrm{S}$ et al. Clinical features suggestive of meningitis in children: a systematic review of prospective data. Pediatrics. 2010;126(5):952-60.

11. Chinchankar $\mathrm{N}$ et al. Diagnosis and outcome of acute bacterial meningitis in early childhood. Indian Pediatr. 2002;39(10):914-21.

12. Tripathy $\mathrm{S}$ et al. Clinicoepidemiological study of viral acute encephalitis syndrome cases and comparison to nonviral cases in children from Eastern India. J Glob Infect Dis. 2019;11(1):7-12.

13. Fitzwater $\mathrm{S}$ et al. Bacterial meningitis in children $<2$ years of age in a tertiary care hospital in South India: an assessment of clinical and laboratory features. J Pediatr. 2013;163(1 Suppl):S32-7.

14. De A et al. Clinical and laboratory profile of acute bacterial meningitis in a tertiary care hospital in Mumbai. IntJ Sci Study. 2016;4(6):113-20.

15. Jayaraman $Y$ et al.; Hospital Based Sentinel Surveillance of Bacterial Meningitis (HBSSBM) Network Team. Burden of bacterial meningitis in India: preliminary data from a hospital based sentinel surveillance network. PloS One. 2018;13(5):e0197198.
16. Kakarlapudi S et al. Comparison of scrub typhus meningitis with acute bacterial meningitis and tuberculous meningitis. Indian Pediatr. 2018;55(1):35-7.

17. Abhilash $\mathrm{K}$ et al. Scrub typhus meningitis: an under-recognized cause of aseptic meningitis in India. Neurol India. 2015;63(2):209-14.

18. Varghese $\mathrm{G}$ et al. Differential diagnosis of scrub typhus meningitis from bacterial meningitis using clinical and laboratory features. Neurol India. 2013;61(1):17-20.

19. Dinesh kumar $\mathrm{N}$ et al. Clinical profile of scrub typhus meningoencephalitis among South Indian children. J Trop Pediatr. 2018;64(6):472-8.

20. Parameswaran K. Case series of eosinophilic meningoencephalitis from South India. Ann Indian Acad Neurol. 2006;9(4):217-22.

21. Joshi $R$ et al. Changing landscape of acute encephalitis syndrome in India: a systematic review. Natl Med J India. 2012;25(4):212-20.

22. Beig $\mathrm{F}$ et al. Etiology and clinicoepidemiological profile of acute viral encephalitis in children of western Uttar Pradesh, India. Int J Infect Dis. 2010;14(2):e141-6.

23. Kumar $\mathrm{R}$ et al. Epidemiological profile of acute viral encephalitis. Indian J Pediatr. 2018;85(5):358-63. 